\title{
A multisite model of allosterism for the adenosine
}

\section{A1 receptor}

Giuseppe Deganutti ${ }^{1, *}$, Kerry Barkan ${ }^{2,8}$, Graham Ladds ${ }^{2}$ and Christopher A. Reynolds ${ }^{1, *}$

${ }^{1}$ Centre for sport, Exercise and Life Sciences, Faculty of Health and Life Sciences, Coventry University, Alison Gingell Building, Coventry, CV1 5FB, UK

${ }^{2}$ Department of Pharmacology, University of Cambridge, Tennis Court Road, Cambridge, CB2 1PD, UK

$\S$ New address: Sosei Heptares, Granta Park Steinmetz Building, Cambridge, CB21 6DG, UK

* Corresponding authors: ad5288@ coventry.ac.uk ; ad5291@ @oventry.ac.uk 


\section{Supplementary Video S1.}

SuMD simulations of PD81723 binding to the (NECA) occupied $\mathbf{A}_{\mathbf{1}} \mathbf{R}$. PD81723 and NECA are shown in van der Waals representation, while the protein residues in its proximity are shown as stick representation. The main hydrogen bonds are highlighted with red dotted lines. The simulation time indicated is cumulative of all the ten replicas. SuMD path sampling was seeded from these SuMD simulations. The frames were smooth to facilitate the visualization.

\section{Supplementary Video S2.}

SuMD simulations of VCP171 binding to the the (NECA) occupied $\mathbf{A}_{1} \mathbf{R}$. VCP171 and NECA are shown in van der Waals representation, while the protein residues in its proximity are shown as stick representation. The main hydrogen bonds are highlighted with red dotted lines. The simulation time indicated is cumulative of all the ten replicas. SuMD path sampling was seeded from these SuMD simulations. The frames were smooth to facilitate the visualization.

\section{Supplementary Video S3}

SuMD simulations of PD81723 binding to the apo $\mathbf{A}_{\mathbf{1}} \mathbf{R}$. PD81723 is shown in van der Waals representation, while the protein residues in its proximity are shown as stick representation. The main hydrogen bonds are highlighted with red dotted lines. The simulation time indicated is cumulative of all the ten replicas. SuMD path sampling was seeded from these SuMD simulations. The frames were smooth to facilitate the visualization.

\section{Supplementary Video S4}

SuMD simulations of VCP171 binding to the apo $\mathbf{A}_{1} \mathbf{R}$. VCP171 is shown in van der Waals representation, while the protein residues in its proximity are shown as stick representation. The main hydrogen bonds are highlighted with red dotted lines. The simulation time indicated is cumulative of all the ten replicas. SuMD path sampling was seeded from these SuMD simulations. The frames were smooth to facilitate the visualization.

\section{Supplementary Video S5}

SuMD simulations of $13 B$ binding to the apo inactive $\mathbf{A}_{1} \mathbf{R}$. 13B is shown in van der Waals representation, while the protein residues in its proximity are shown as stick representation. The main hydrogen bonds are highlighted with red dotted lines. The simulation time indicated is cumulative of all the ten replicas. SuMD path sampling was seeded from these SuMD simulations. The frames were smooth to facilitate the visualization. 


\section{Supplementary Video S6}

SuMD simulations of VCP746 binding to the apo $\mathbf{A}_{\mathbf{1}} \mathbf{R}$. VCP746 is shown in van der Waals representation, while the protein residues in its proximity are shown as stick representation. The main hydrogen bonds are highlighted with red dotted lines. The simulation time indicated is cumulative of all the ten replicas. SuMD path sampling was seeded from these SuMD simulations. The frames were smooth to facilitate the visualization.

\section{Supplementary Video S7}

SuMD simulations of NECA binding to the (VCP171) occupied $\mathbf{A}_{1} \mathbf{R}$. VCP171 and NECA are shown in van der Waals representation, while the protein residues in its proximity of NECA are shown as stick representation. SuMD path sampling was seeded from these SuMD simulations. The frames were smooth to facilitate the visualization. 


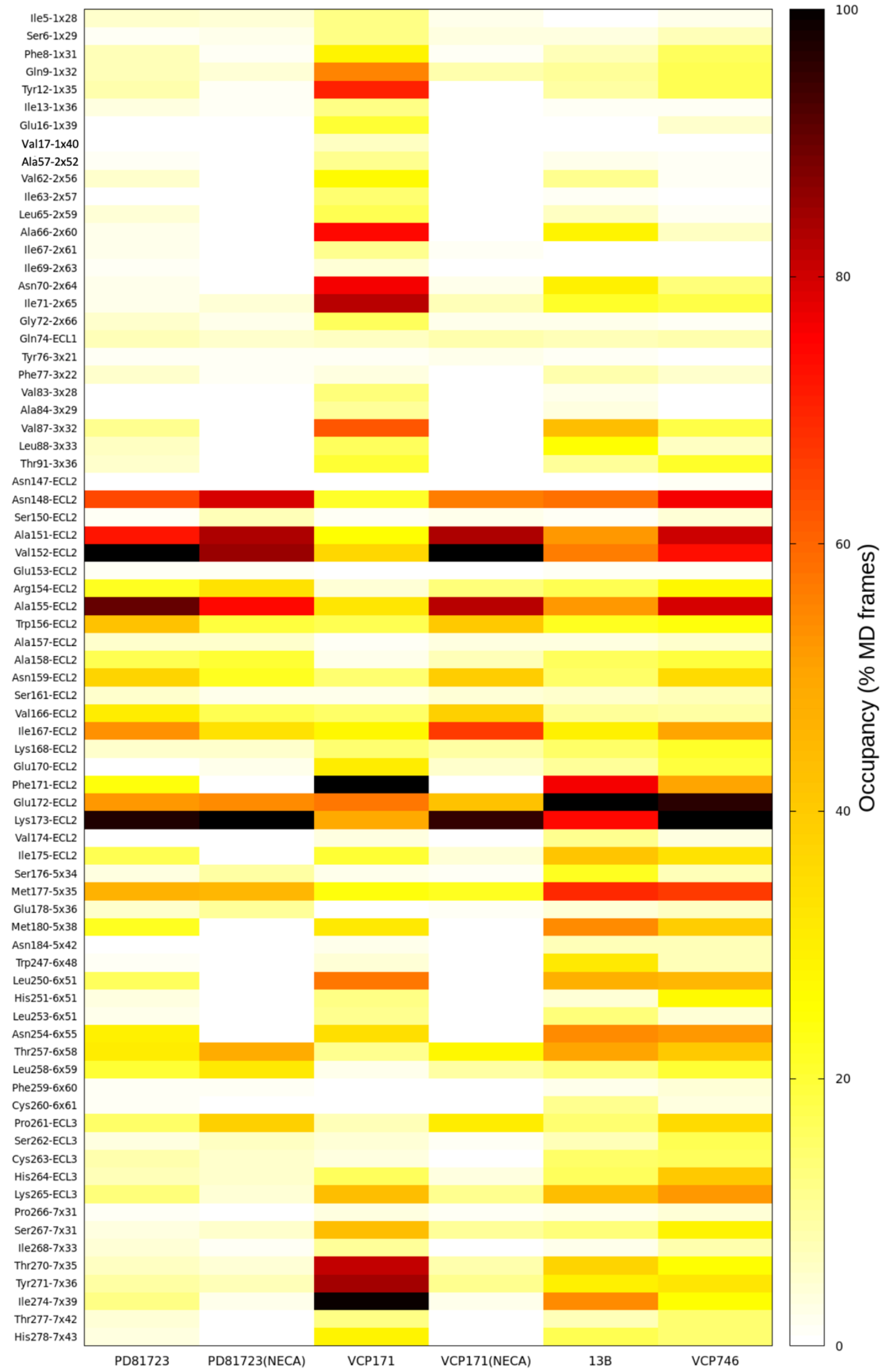

Figure S1. Ligands - $A_{1} R$ contacts heatmap. For each $A_{1} R$ residue in each system, the total contact occupancy during the simulations (\% MD frames in which the interaction occurred) is normalized for the highest occupancy value. 

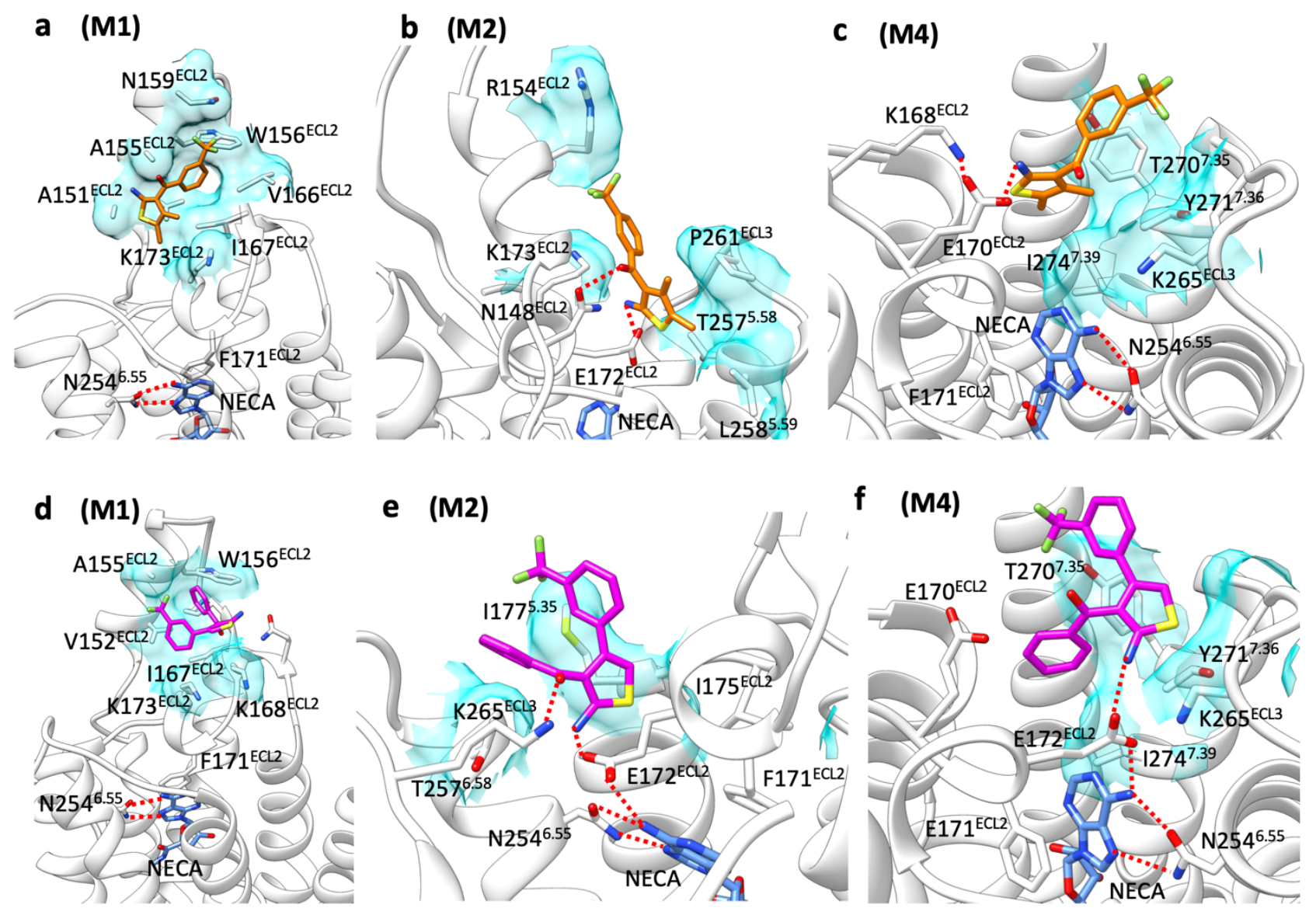

Figure S2. Representative complexes (microstates) from PD81723 and VCP171 SuMD binding simulations of the occupied $A_{1} R$, according to MM-GBSA analysis. a) - c) representative configurations from PD81723 (orange stick) metastable macrostates M, M2, and M4 in presence of NECA (blue stick) in the orthosteric site; d) - f) representative configurations from VCP171 (magenta stick) metastable macrostates M1, M2, and M4 in presence of NECA (blue stick) in the orthosteric site. Hydrophobic contacts are shown as transparent cyan surfaces, hydrogen bonds are reported as dashed red lines. 

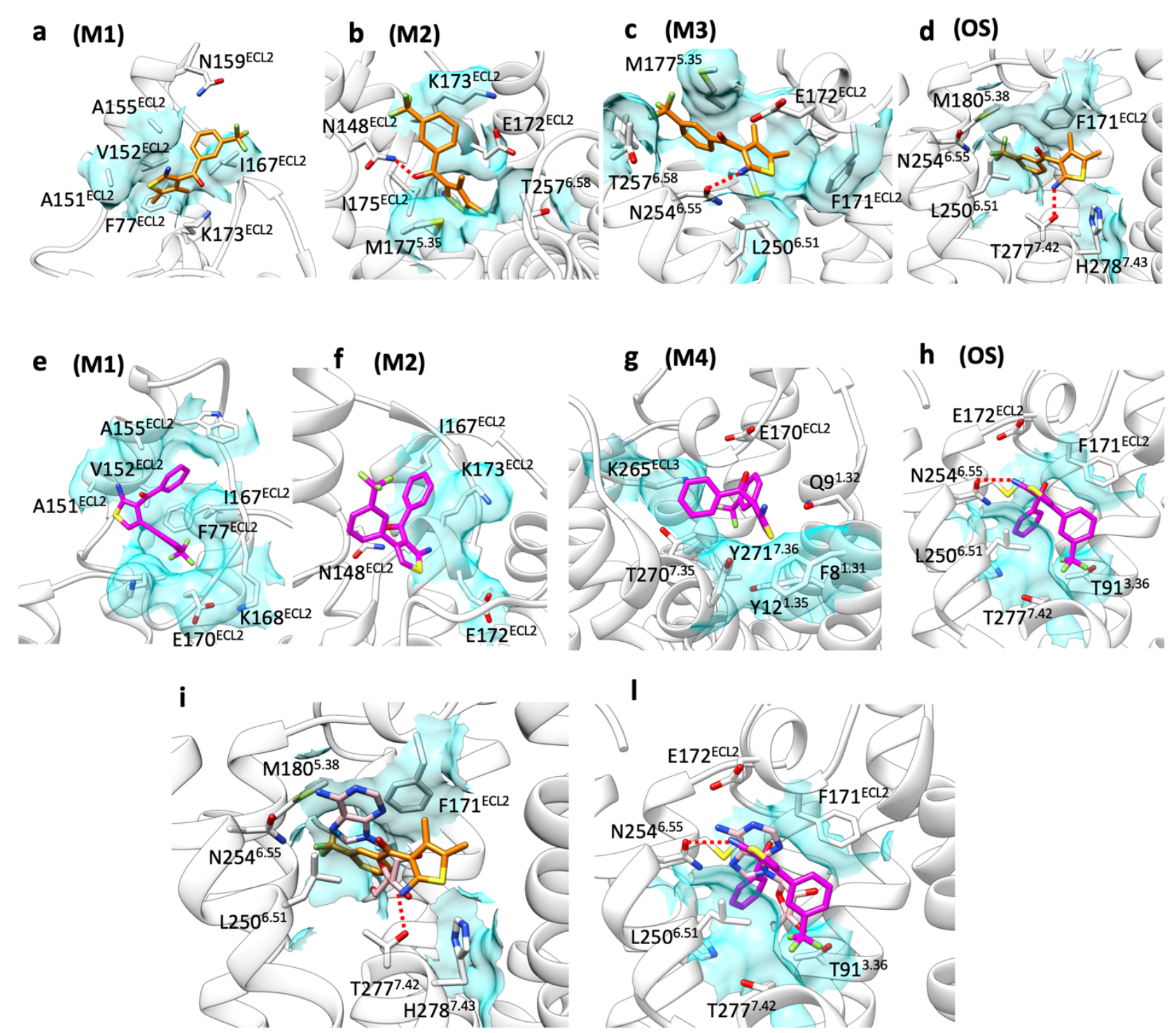

Figure S3. Representative complexes (microstates) from PD81723 and VCP171 SuMD binding simulations on the unoccupied $A_{1} R$, according to MM-GBSA analysis. a) - d) representative configurations from PD81723 (orange stick) metastable macrostates M1-M3 and the bound state OS; d) - h) representative configurations from VCP171 (magenta stick) metastable macrostates M1, M3, M4 and the bound macrostate OS. i) superposition between PD81723 (OS) and adenosine (cryo-EM structure, pink); l) superposition between VCP171 (OS) and adenosine (cryo-EM structure, pink). Hydrophobic contacts are shown as transparent cyan surfaces, hydrogen bonds are reported as dashed red lines; in h) TM6 residues are not shown. 


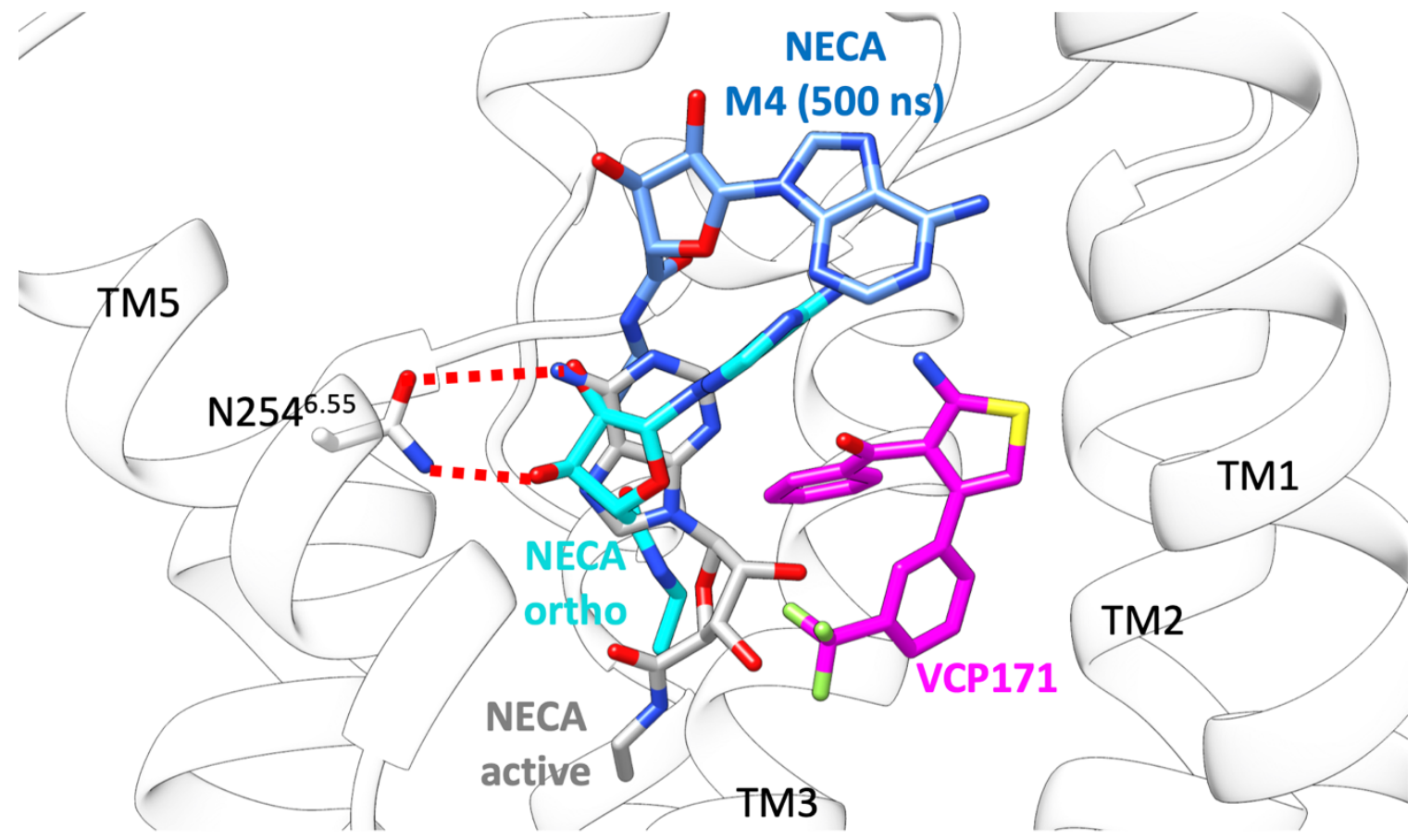

Figure S4. NECA:VCP171: $A_{1} R$ ternary complex formed during SuMD simulations. In several SuMD replicas NECA reached the VCP171-occupied orthosteric site. During three classic MD replicas (500 ns each) started from one of configuration showed (NECA in cyan, VCP171 in magenta) NECA moved to the macrostate M4 (blue), above VCP171. NECA active bound conformation is reported in light grey. Hydrogen bonds between N254 $4^{6.55}$ and NECA in the ternary complex are shown as dashed red lines.

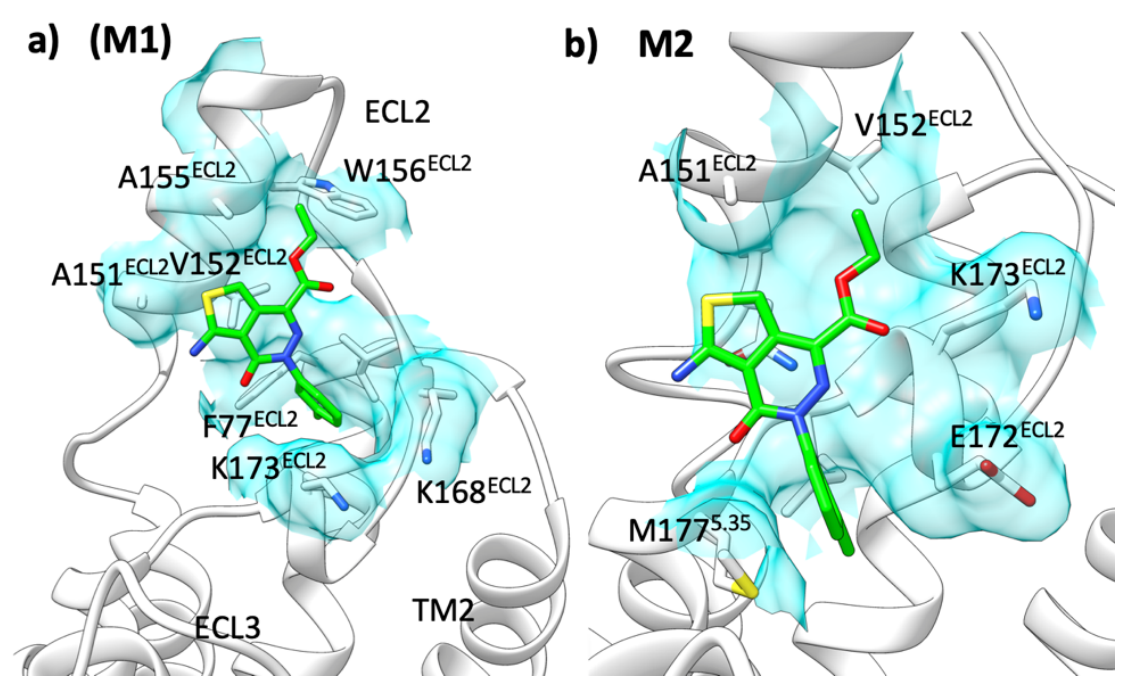


Figure S5. Representative complexes (microstates) from compound 13B SuMD binding simulations on the unoccupied inactive $A_{1} R$, according to MM-GBSA analysis. a) Microstate from PD81723 (green stick) metastable macrostates M1; b) Microstate from PD81723 (green stick) metastable macrostates M2. Hydrophobic contacts are shown as transparent cyan surfaces.
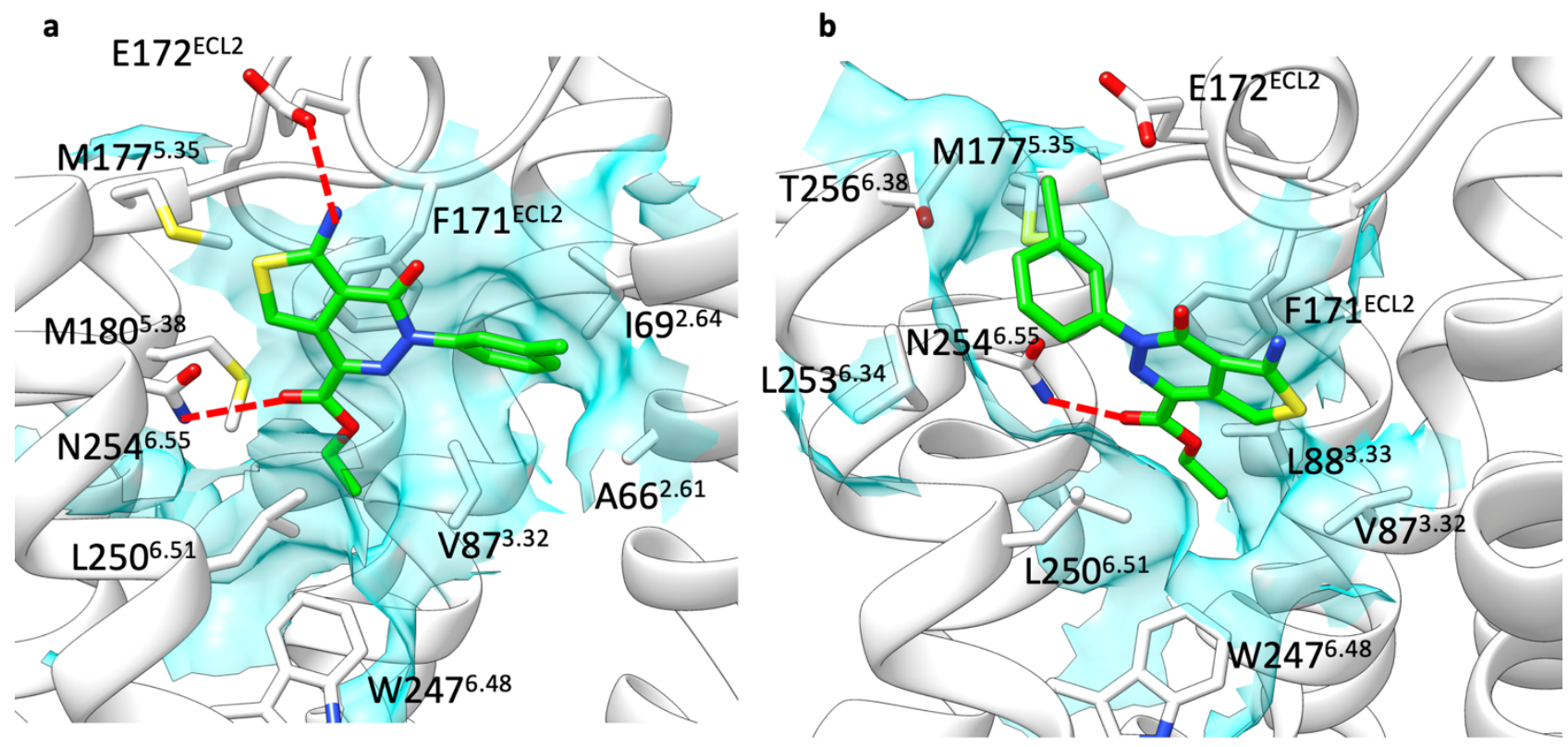

Figure S6. Representative complexes (microstates) from compound 13B SuMD binding simulations on the unoccupied inactive $A_{1} R$, according to MM-GBSA analysis. a) and b) Microstates from PD81723 (green stick) orthosteric macrostate OS. Hydrophobic contacts are shown as transparent cyan surfaces, hydrogen bonds are reported as dashed red lines. 
a

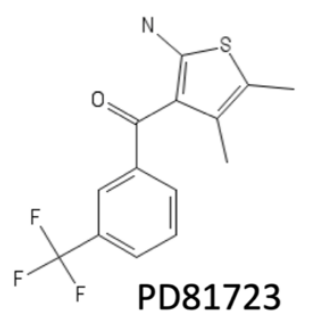

PD81723
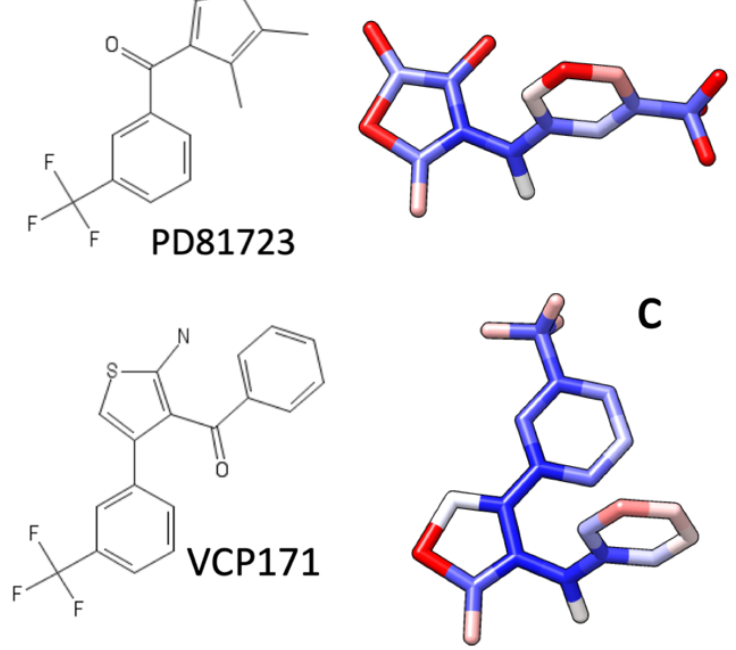

C

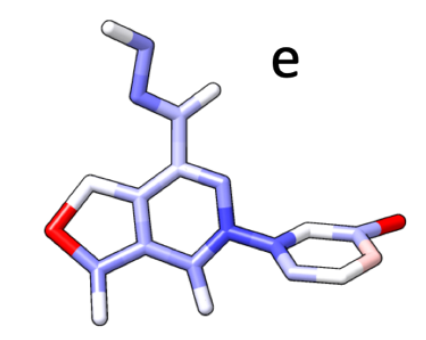

$13 B$
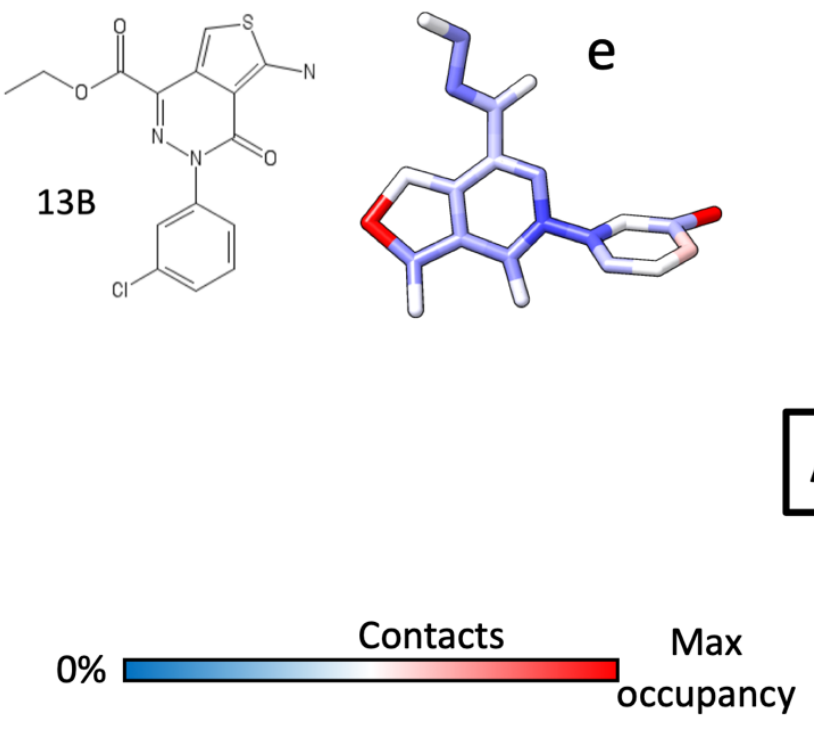

b
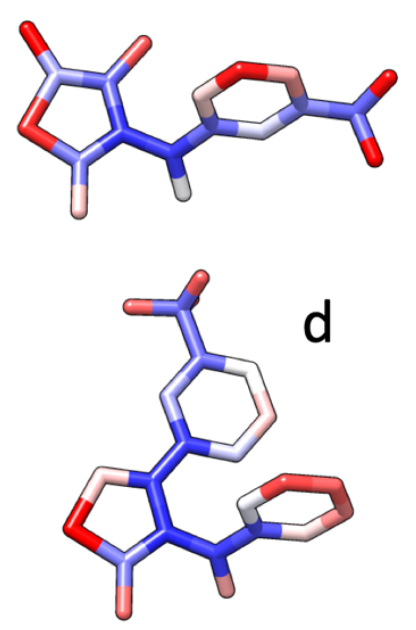

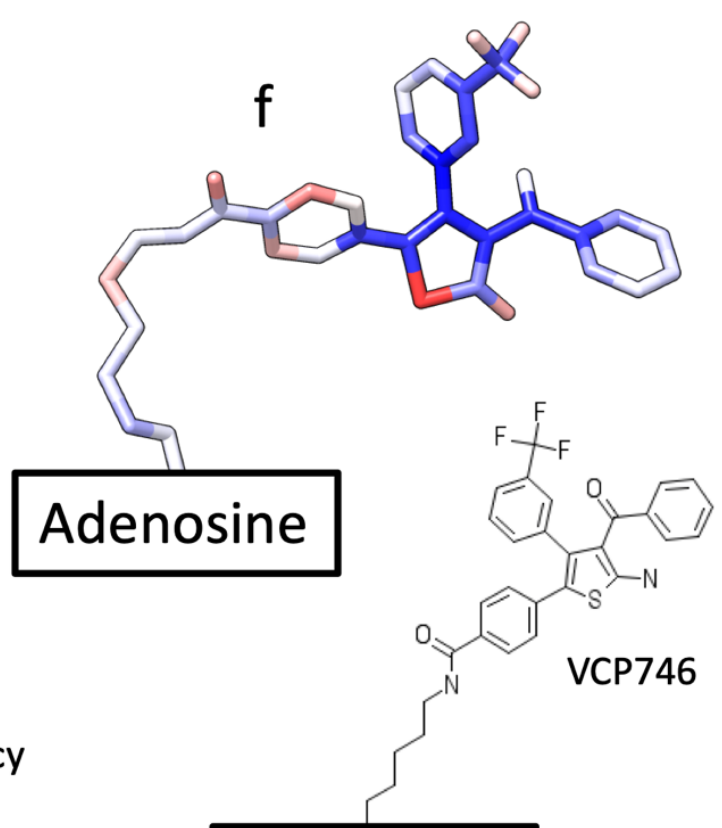

Adenosine

Figure S7. Ligands' atoms involved in contacts with $A_{1} R$ during SuMD simulations. a) PD81723-occupied $A_{1} R$; b) PD81723-unoccupied $A_{1} R$; c) VCP171-occupied $A_{1} R$; d) VCP171occupied $A_{1} R$; e) Compound 13B-unoccupied inactive $A_{1} R ; f$ ) VCP746-unoccupied $A_{1} R$. Atoms are colored according to the total percentage of MD frames (occupancy) in which they were involved in contacts with the receptor. Among the simulated ligands, compound 13B was the less involved at the level of the 2-aminogroup. 


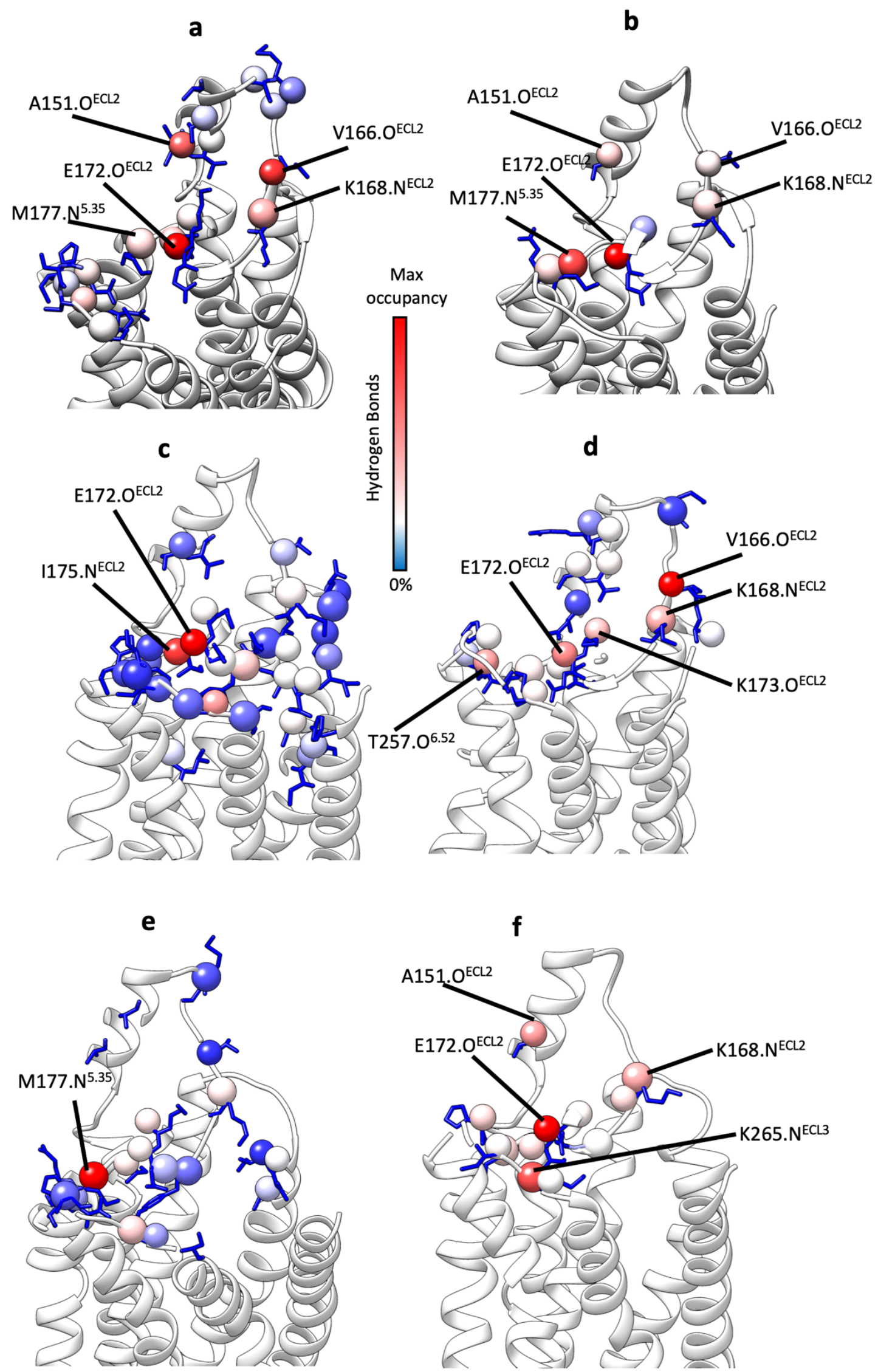

Figure S8. Main $\mathrm{A}_{1} \mathrm{R}$ backbone atoms involved in hydrogen bonds during simulations. a) PD81723occupied $\mathrm{A}_{1} \mathrm{R}$ (NECA bound to the orthosteric site); b) PD81723-unoccupied $\mathrm{A}_{1} \mathrm{R}$; $\mathbf{c}$ ) VCP171- 
occupied $A_{1} R$ (NECA bound to the orthosteric site); d) VCP171-unoccupied $A_{1} R$; $\mathbf{e}$ ) compound 13Bunoccupied inactive $\left.A_{1} R ; \mathbf{f}\right) C P 746-$ unoccupied $A_{1} R$.

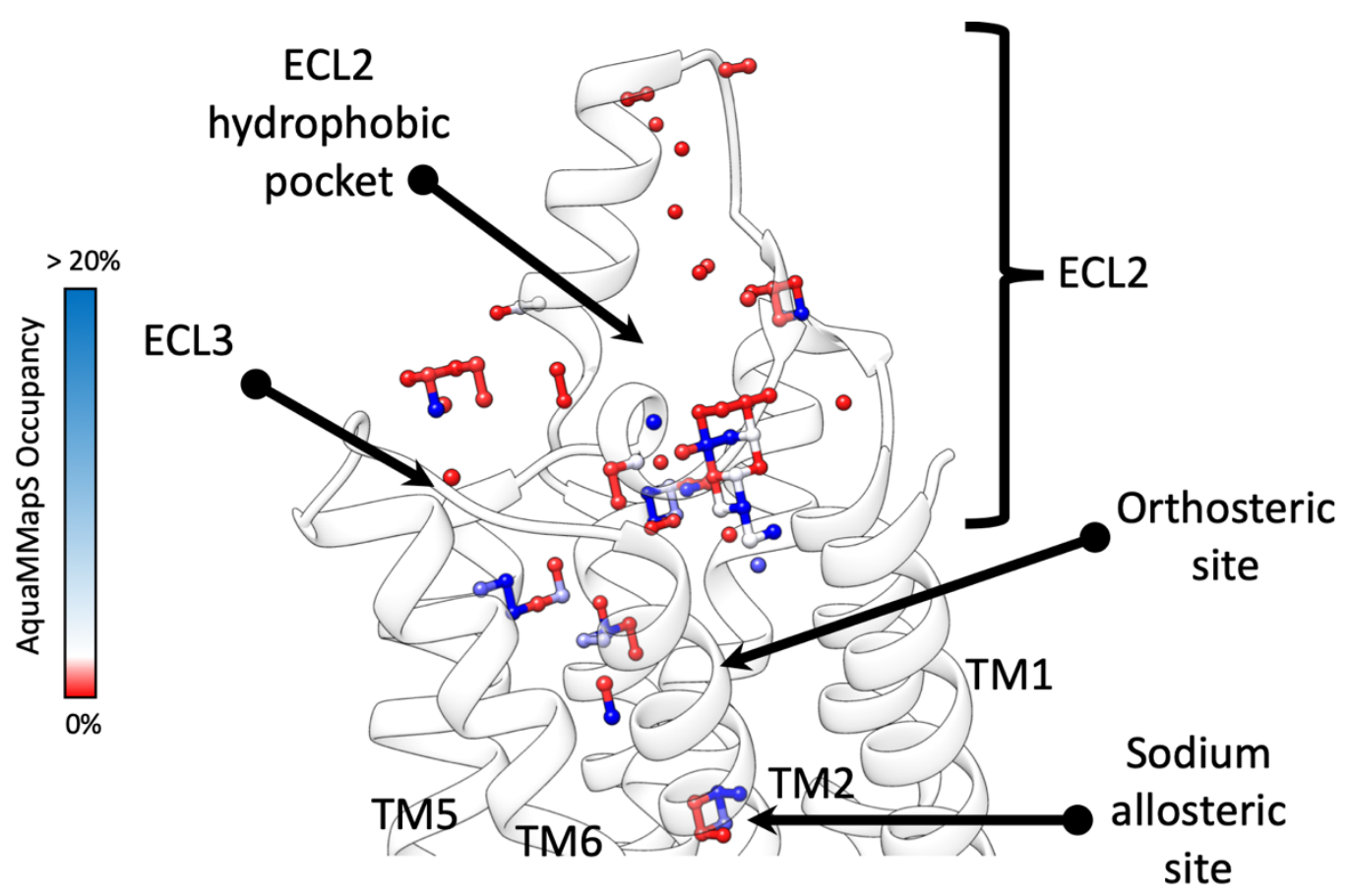

Figure S9. Hydrated spots on the $\mathrm{A}_{1} \mathrm{R}$ surface (within $4 \AA$ of backbone atoms) according to AquaMMaPS analysis. Beside the allosteric site for the sodium, further region characterized by stabilized water molecules occurred in the orthosteric site and at the ECL2 and ECL3. The hydrophobic pocket within ECL2 (responsible for stable states M1 in Figure 2, Figure 3, Figure S2, Figure S3) shows low tendency to stable hydration. 


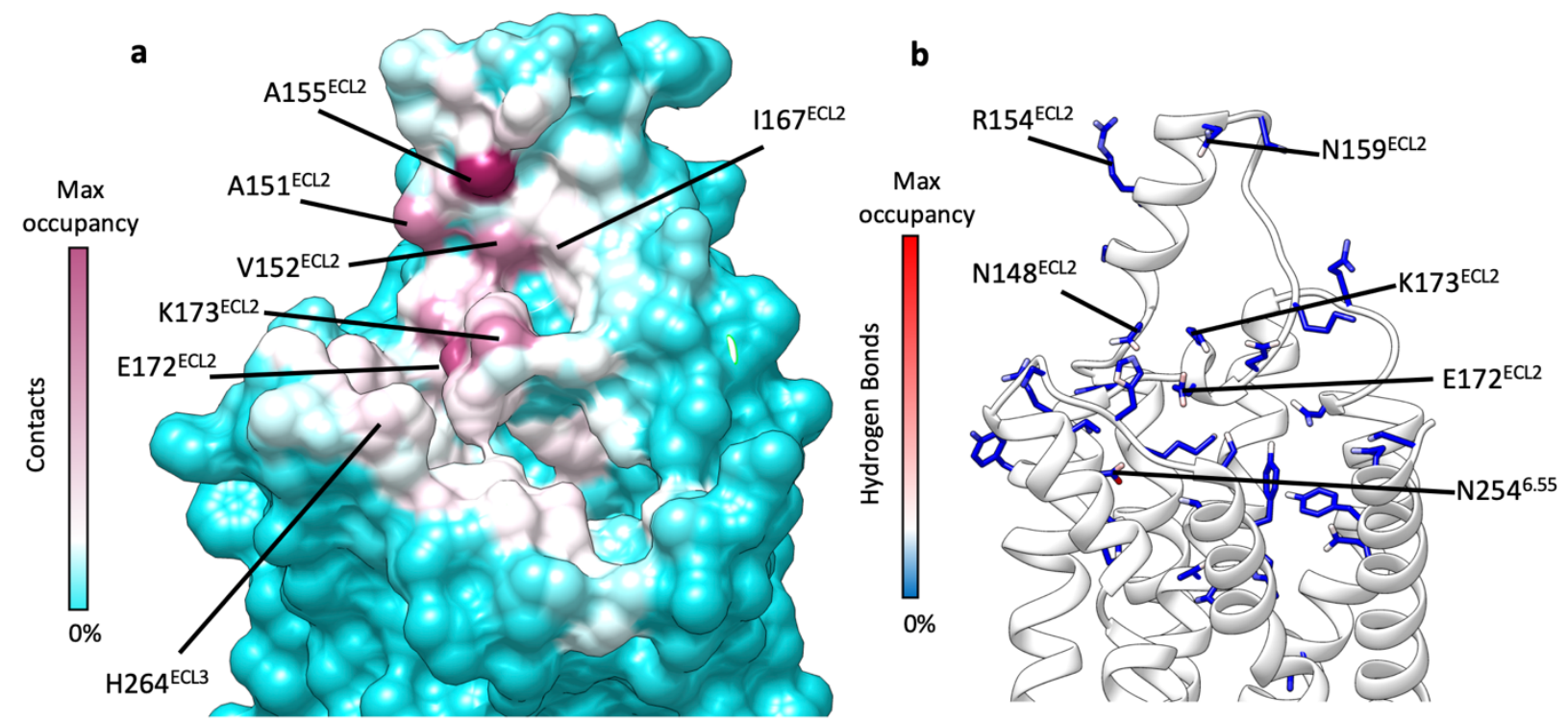

Figure S10. a) $A_{1}$ R-VCP746 contacts plotted on the receptor surface and colored according to the occupancy (\% MD frames in which a contact was present); b) $\mathrm{A}_{1} \mathrm{R}$ side chain atoms that formed hydrogen bonds with VCP746 and colored according to the occupancy (\%MD frames in which a hydrogen bond was present). 